\title{
Создание и использование третьей гармоники лазерного генератора на 355 нм для участия в эксперименте ТУС
}

\author{
А.И. Дмитроциа, И.В. Артёмов, Д.И. Неяченко \\ ФГБУН “Крымская астрофизическая обсерватория РАН”, Научный, Крым, 298409 \\ dmytrotsa@gmail.com
}

Поступила в редакцию 15 ноября 2017 г.

\begin{abstract}
Аннотация. Для калибровки детектора на орбите в качестве одного из вариантов предполагается использование лазерного излучения на третьей гармонике (355 нм) твердотельного лазера "ЛСД Simeiz 1873”. Как показано в работе (Городецкий и др., 2011), лазерный спутниковый дальномер можно использовать как источник ультрафиолета для калибровки.

На станции успешно реализована генерация третьей гармоники и проводятся регулярные эксперименты по калибровке ТУС в космосе. Для расширения возможностей экспериментов была разработана и внедрена новая уточненная модель ошибок телескопа.

CREATION AND USAGE OF THE THIRD HARMONIC OF THE LASER GENERATOR AT 355 NM FOR PARTICIPATION IN THE TUS EXPERIMENT, by A.I. Dmitrotsa, I.V. Artyomov, D.I. Neyachenko. To calibrate the on-orbit detector, as one of variants, it is supposed to use laser beam at the third harmonic of the solid-state laser in the Satellite Ranging Station (SLR) "Simeiz-1873". As is shown by (Gorodetzky et al., 2011), the SLR is to be used as an ultraviolet source for calibration.

The third harmonic generation has been successfully implemented at the station and regular experiments on calibrating TUS in space are being carried out. To expand opportunities for experiments, a new model of telescope errors has been elaborated and introduced.
\end{abstract}

Ключевые слова: ТУС, космический детектор, лазерный спутниковый дальномер, ультрафиолет

\section{1 Введение}

Спутник “Ломоносов” был запущен 16 апреля 2016 года. Это целая космическая лаборатория, которая должна изучать самые экстремальные явления во Вселенной - космические лучи предельно высоких энергий (КЛПВЭ, $E \sim 10^{19}-10^{20}$ эВ), гамма-всплески и воздействие энергичных частиц в околоземном космическом пространстве на земную атмосферу. В настоящее время он находится на орбите и полностью функционирует.

Орбитальный телескоп ТУС (Трековая УСтановка) на спутнике “Ломоносов” - это первый в мире инструмент, предназначенный для регистрации треков от КЛПВЭ в атмосфере Земли с борта космического аппарата. Он регистрирует следы космических частиц - быстрые ультрафиолетовые (УФ) вспышки, возникающие при взаимодействии каскада вторичных частиц от КЛПВЭ с атомами воздуха на высотах в десятки километров. 


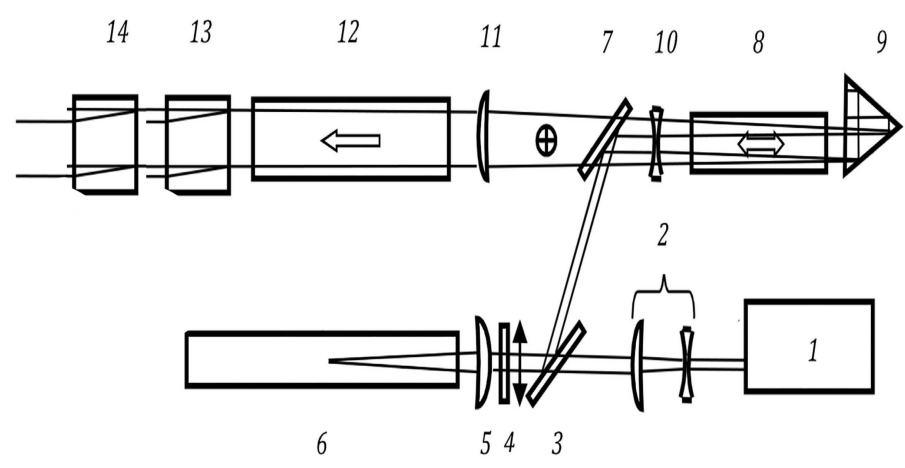

Рис. 1. Оптическая схема лазерного излучателя с компрессором на основе эффекта вынужденного рассеяния Мандельштама-Бриллюена (ВРМБ) с двухкаскадным усилителем. 1 - задающий генератор; $2,10,11$ расширяющие телескопы; 3, 7 - диэлектрические поляризаторы; 4 - четвертьволновая пластинка; 5 - линза компрессора; 6 - ВРМБ-кювета; 8- усилитель первого каскада; 9 - призменный фазовращатель; 12 - усилитель второго каскада, 13 - кристалл DKDP, 14 - кристалл KTP

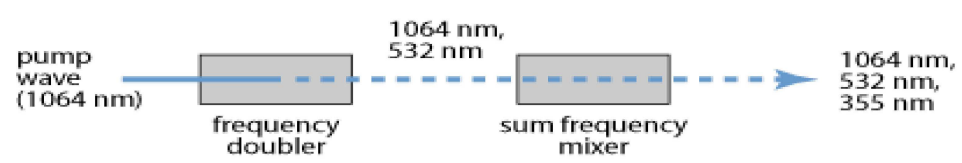

Рис. 2. Схема получения третьей гармоники на нелинейных кристаллах $\operatorname{DKDP}\left(K_{2} D_{2} O_{4}\right)$ и $\mathrm{KTP}\left(K T i O P O_{4}\right)$

Для эксперимента ТУС планируется разработать и создать наземную систему источников света в УФ для калибровки в полете ТУС-детектора. Для этой цели в настоящее время рассматриваются два типа возможных источников УФ-излучения: светодиоды и лазерные станции.

Представлены конструкция и изготовление прототипов источника калибровочного излучения на основе лазерной станции “Simeiz-1873”. На первом этапе преобразования передатчика необходимо внести изменения в оптическую схему лазерного излучателя (рис. 1).

Чтобы обеспечить необходимый УФ-диапазон, требуется ввести генератор третьей гармоники в схему излучателя. Для получения третьей гармоники использовались свойства нелинейных кристаллов, таких как $\operatorname{DKDP}\left(K_{2} \mathrm{PO}_{4}\right)$ и $\mathrm{KTP}\left(\mathrm{KTiOPO}_{4}\right)$ (рис. 2).

Кроме того, оптика телескопа дальномера также должна быть адаптирована к УФ-диапазону. Для этого потребовалось заменить некоторые зеркала с покрытием на 532 нм на зеркала с широкополосным покрытием.

Как показали первые результаты, требуется уделять особое внимание методам ведения спутника и регистрации момента калибровки. Из-за особенностей орбиты и узкого поля зрения прибора ТУС возможное время его калибровки ограничено буквально десятком секунд.

\section{2 Экспериментальная калибровка детектора ТУС}

Главным преимуществом использования станции лазерной локации спутников для этой работы, безусловно, является наличие мощного источника излучения и управляемого телескопа. Это позволяет получить нужную энергию на определенной длине волны и направить ее в необходимый участок неба. Для проведения эксперимента рассмотрено два варианта: 
1. Базовый вариант. Направляя луч под определенным углом, добиться появления в атмосфере рассеивания от УФ-луча. Этот луч, согласно расчетам (Городецкий и др., 2011), будет виден со спутника, и его интенсивность будет близка к изучаемым следам в атмосфере космических лучей сверхвысоких энергий.

2. Дополнительный вариант. Наблюдение спутника одновременно на длинах волн 355 и 532 нм. Это позволяет получать как расстояния до спутника (для определения и уточнения его орбиты), так и измерения отражения УФ от элементов спутника. Это важно для учета бликов от ярких далеких событий.

Как показал опыт, из-за большой чувствительности прибора ТУС, триггер очень часто срабатывает на случайные события. Например, во время эксперимента 2017-07-21 триггер сработал в северной части Крыма, буквально за несколько секунд до подлета к нашей станции. А вот во время эксперимента 2017-09-07, как выяснилось позднее, оборудование на спутнике было полностью отключено из-за сильнейшей вспышки на Солнце.

При нахождении спутника в тени корректировка положения телескопа оператором невозможна, и от телескопа требуется точность наведения с ошибкой порядка $20^{\prime \prime}$. При идеальных монтировках, для построения модели ошибок телескопа с нужной точностью, достаточно простых математических моделей и относительно небольшого количества измерений положений звезд.

K сожалению, после изучения нашего телескопа выяснилось, что он далеко не идеален. У телескопа наблюдаются биения, вызванные механической частью монтировки. Это приводит к тому, что простыми моделями описать телескоп не удается. Усугубляют ситуацию и относительные угловые датчики, приводящие к неопределенности положения нулевой точки телескопа.

Чтобы решить эту задачу, нужно построить точную модель ошибок телескопа (Нестеров, 1985). Для этого необходимо большое количество измерений положений звезд. Но в (Медведский, Пап, 2008) был предложен вариант использования измерений положения спутников во время обычных наблюдений. Это позволяет, не прекращая наблюдений, накапливать измерения положений для построения модели.

Имея порядка 12000 измерений положений, на текущий момент мы добились модели со среднеквадратичным отклонением $60^{\prime \prime}$. Этого недостаточно. Требуется дальнейший набор статистики по положениям, и, возможно, усложнение модели для отработки сложного характера ошибок.

\section{3 Заключение}

Мы создали работающий элемент калибровки аппарата ТУС на базе станции “Simeiz-1873”. Проводим регулярные эксперименты по калибровке детектора ТУС. Для расширения возможностей станции была разработана модель ошибок телескопа со среднеквадратичным отклонением $60^{\prime \prime}$. В идеале необходимо добиться слепого ведения спутников.

\section{Литература}

Городецкий и др. (Gorodetzky P., Sakaki N., Christl M.) // Proceedings of the 32nd International Cosmic Ray Conference / Ed. Suresh Tonwar. Beijing: IHEP. 2011. V. 3. P. 36.

Медведский, Пап (Medvedskiy M., Pap V.) // Proceedings of the 16th International Workshop on Laser

Ranging / Ed. Schillak S. Poznań: Space Research Centre, PAS. 2008. P. 483.

Нестеров (Nesterov N.S.) // Bull. Crimean Astrophys. Obs. 1985. V. 73. P. 169. 